\title{
Study of collectivity in $p+p$ collisions at LHC energies.
}

\section{Jajati K. Nayak}

Variable Energy Cyclotron Centre

E-mail: jajati-quark@vecc.gov.in

Relativistic $p+p$ collisions at LHC energies shows some striking observations which ignite intense debate. Countering the argument they serve as the base line for the analysis of heavy ion collisions, a different view has emerged that they produce a medium like heavy ion collisions with a smaller size. The charge hadron spectra produced from $p+p$ collisions at $\sqrt{s_{N N}}=0.9,2.76$ and $7 \mathrm{TeV}$, LHC energies have already been analysed on the basis of blast-wave analysis [1] to review the argument of collectivity and medium formation. Here we present the results for the spectra of identified particles $(\pi, K, p)$ at $7 \mathrm{TeV} p+p$ collisions from the hydrodynamical analysis. The spectra have been evaluated for different mean multiplicities and compared with the data measured by CMS collaboration [2].

7th International Conference on Physics and Astrophysics of Quark Gluon Plasma

1-5 February, 2015

Kolkata, India 


\section{Introduction}

The possibility of formation of a collective partonic medium, called Quark Gluon Plasma (QGP) in relativisitc $p+p$ collisions is a matter of intense debate in recent days. Several theoretical activities [3,4] with different analysis are available by now. At the Large Hadron Collider(LHC), there is an order of magnitude increase in the colliding energies compared to those available at Super Proton Synchrotron (SPS) and Relativistic Heavy Ion Collider(RHIC). The increase in energy by such an amount provoked to think about the formation of QGP.

The study of QGP formation in relativistic $p+p$ collisions at LHC, is not only because of the argument of increasing colliding energy, also because of some striking experimental observations. For example, the formation of ridge like structure which has been observed by CMS collaboration at high multiplicity events at $\sqrt{s_{N N}}=7 \mathrm{TeV}$, LHC energy[2], is quite similar in nature to the one observed at RHIC heavy-ion collisions [7]. The presence of such ridge structure has already been argued to arise due to the formation of hydrodynamical system. The measurements from ALICE and CMS in [5, 6] show the dependencies of HBT-radii on multiplicity and pair transverse momentum $\left(p_{T}\right)$ which might be a feature of hydrodynamical behavior. Similar kind of behavior is also observed in heavy ion collisions at RHIC [8]. Here we study the collectivity in $p+p$ collisions at $\sqrt{s_{N N}}=7 \mathrm{TeV}$, at LHC using hydrodynamics and with an equation of state that mimics the lattice predicted equation of state at high temperature $\left(>T_{t r}\right)$ and the Hadronic Resonance Gas Model equation of state at low temperature $\left(<T_{t r}\right)$. The successful application of hydrodynamics by explaining the experimental observations implies the presence of collectivity in the medium.

In the next section we discuss the hydrodynamics and the production of chage particle spectra from $p+p$ collisions with a brief summary.

\section{Hydrodynamic description of charge particle spectra}

We assume that the produced system achieve thermalisation (local) and expands due to high internal pressure following the equation of ideal hydrodynamics, $\partial_{\mu} T^{\mu v}=0$. Where $T^{\mu v}=(\varepsilon+$ $P) u^{\mu} u^{v}-g^{\mu v} P$ is the energy-momentum tensor for ideal fluid and $\varepsilon, P$ are the energy and pressure densities respectively. $g^{\mu v}=\operatorname{diag}(1,-1,-1,-1)$ is the metric tensor and $u^{\mu}$ is the fluid four velocity. The equation is solved assuming longitudinal boost invariance [10] and cylindrical symmetry. To solve this boundary value problem we have assumed the initial central energy density and initial time (thermalisation time). To explain the charge particle $p_{T}$ spectra for $\sqrt{s_{N N}}=7 \mathrm{TeV}$, the initial energy density, $\varepsilon\left(\tau_{i}, r\right)$ and radial velocity, $v_{r}\left(\tau_{i}, r\right)$ profiles are taken as: $\varepsilon\left(\tau_{i}, r\right)=\frac{\varepsilon_{0}}{1+e^{\frac{r-R_{A}}{\delta}}}$ and $v_{r}\left(\tau_{i}, r\right)=v_{0}\left(1-\frac{1}{1+e^{\frac{r-R_{A}}{\delta}}}\right), \varepsilon_{0}$ is the initial (central) energy density. The surface thickness, $\delta=0.5 \mathrm{fm}$ and $v_{0}$ is assumed to be zero. The equation of state (EOS) is another important input to the hydrodynamics. Here we consider an ansatz equation of state constructed from both parametrised lattice results and hadronic resonance gas (HRG) model. This EOS has been successful in explaining the photon $\left(p_{T}\right)$ and dilepton ( $p_{T}$ and invariant mass) spectra from heavy ion collisions for wide range colliding energies $[11,12,13,14,15]$ Here, the entropy $\left(s_{q}\right)$ of the QGP phase for $T>T_{t r}$ is considered from lattice EOS and the entropy $\left(s_{h}\right)$ of the hadronic phase is considered from the hadron gas (assuming non-interacting hadrons and their resonances up to 
mass $\sim 2.5 \mathrm{GeV}$ ) for $T<T_{t r}$. The entropy density during transition region is parametrized with a tan-hyperbolic function which can make the transition first order or crossover depending on the value of the width parameter as follows [16],

$$
\begin{array}{r}
s(T)=s_{q}(T) f_{q}(T)+\left[1-f_{q}(T)\right] s_{h}(T) \\
f_{q}(T)=\frac{1}{2}\left(1+\tanh \frac{T-T_{t r}}{\Gamma}\right)
\end{array}
$$

$\Gamma$ is the width parametre, taken to be $20 \mathrm{MeV}$ here. For our calculation $T_{t r}$ is taken to be 175 $\mathrm{MeV}$ [17].

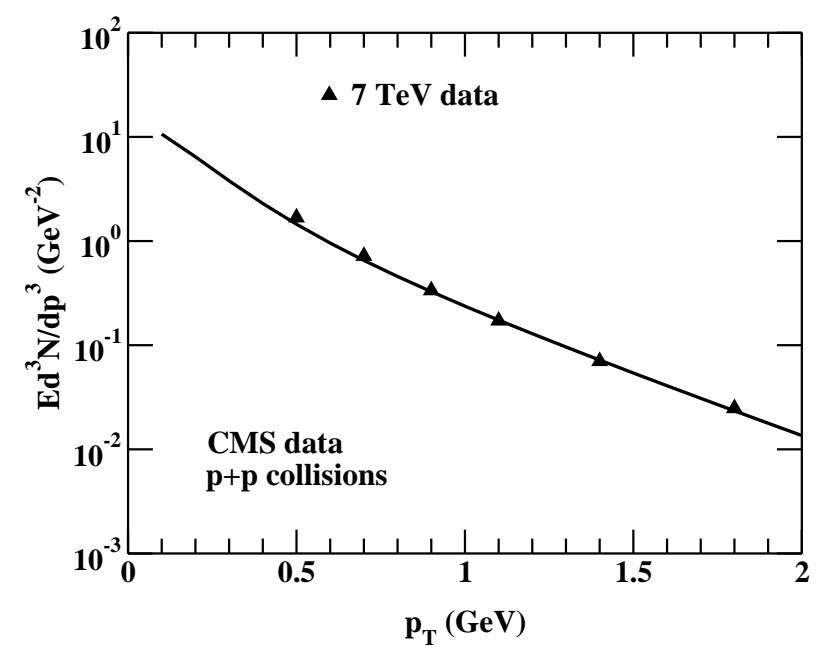

Figure 1: The transverse momentum $\left(p_{T}\right)$ spectra of charge particles have been plotted for $\sqrt{s_{N N}}=7 \mathrm{TeV}$, LHC energy. The solid circles are the data points observed by CMS collaboration [18]. The solid line represents the theoretical calculations.

\subsection{Cooper-Fry Formalism}

In a hydrodynamical point of view, the hadrons in the nuclear collisions may be emitted after decoupling suddenly from the medium at a freeze out hyper surface. Before sudden decoupling the particles might have a hydrodynamic behavior following the above equation of state. The invariant momentum distribution of particles produced at freeze out surface is evaluated using Cooper-Frye prescription [19] as given below.

$$
\frac{E d^{3} N}{d p^{3}} \equiv \int d \sigma_{\mu} p^{\mu} f\left(p^{\mu}, T\right)
$$

$\sigma$ is the sharp freeze out hyper surface and characterized by a constant freeze out temperature $T_{F} . p^{\mu}$ is the 4-momentum and $f$ is the thermal distribution function. The parameters used for the evaluation of the charge particle spectra are mentioned in the next section. 


\subsection{Charged particle spectra}

The $p_{T}$ spectra for charge hadrons from $p+p$ collisions at $\sqrt{s_{N N}}=7 \mathrm{TeV}$ have been evaluated from the hydrodynamic calculations using Cooper-Fry prescription and compared with the experimental observations [18] made by CMS collaborations. This has been shown in Fig. 1. The theoretical estimation is with initial energy density $\varepsilon_{i}=1.55 \mathrm{GeV} / \mathrm{fm}^{3}$ and $\tau_{i}=0.8 \mathrm{fm}$. It explains reasonably well up to $p_{T}=2 \mathrm{GeV}$.

The identified charge particle spectra $\left(\pi^{+}, \pi^{-}, K^{+}, K^{-}, p, \bar{p}\right)$ from $p+p$ collisions at $\sqrt{s_{N N}}=7$ $\mathrm{TeV}$ have been calculated for mean multiplicities 63 and 75 and are shown in Figs. 2 and Fig. 3.

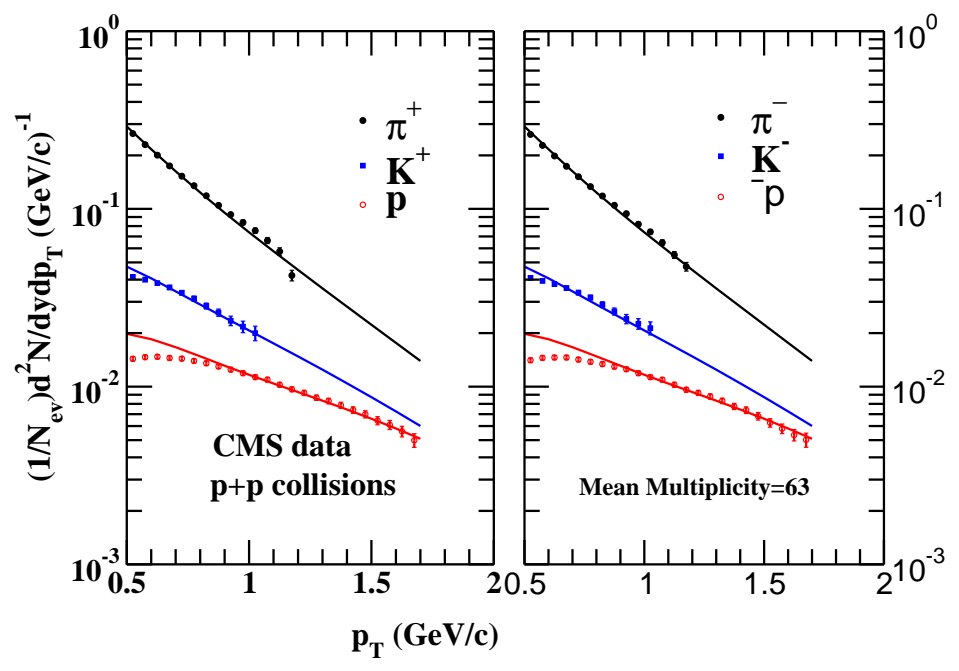

Figure 2: The transverse momentum spectra of identified charged particles such as pions, kaons and protons with mean multiplicity $=63$ have been plotted for $\sqrt{s_{N N}}=7 \mathrm{TeV}$, LHC energy. The solid circles represent the data points observed by CMS collaboration. Here, the left panel is for $\pi^{+}, K^{+}, p$ and the right panel is for $\pi^{-}, K^{-}$and $\bar{p}$. The solid lines are theoretical calculations.

The solid circles (black-color online) both in right and left panels represent the experimental data of $\pi^{+}$and $\pi^{-}$[18] respectively. The solid squares (blue:color online) are for $K^{+} \& K^{-}$and open circles (red: color online) are for $p$ and $\bar{p}$. The solid curves are theoretical estimations using hydrodynamics with initial energy densities $\varepsilon_{0}=1.5 \mathrm{GeV} / \mathrm{fm}^{3}$ (for multiplicity 63 ) and $\varepsilon_{0}=1.6 \mathrm{GeV} / \mathrm{fm}^{3}$ (for multiplicity 75). The initial thermalisation time $\tau_{i}=0.9 \mathrm{fm}$ is considered for both. To explain the pion and kaon spectra we have used the freeze out temperature $T_{F}=115 \mathrm{MeV}$. For proton spectra, both calculations for $T_{F}=115 \mathrm{MeV}$ and $T_{F}=120 \mathrm{MeV}$ are shown. The solid curve (for proton spectra) is for $T_{F}=120 \mathrm{MeV}$ and dashed line is for $T_{F}=115 \mathrm{MeV}$ i.e., for multiplicity=75. Considering the pions and kaons to be emitted from a constant freeze out surface at freeze out temperature $T_{F}=115 \mathrm{MeV}$, the hydrodynamical calculation explains the data reasonably well. However the proton spectra have been explained better with $T_{F}=120 \mathrm{MeV}$ and stil the deviation exists at very low $p_{T}\left(0.5 \leq p_{T}(\mathrm{GeV}) \leq 0.8\right)$. A viscous hydrodynamics may explain well. 


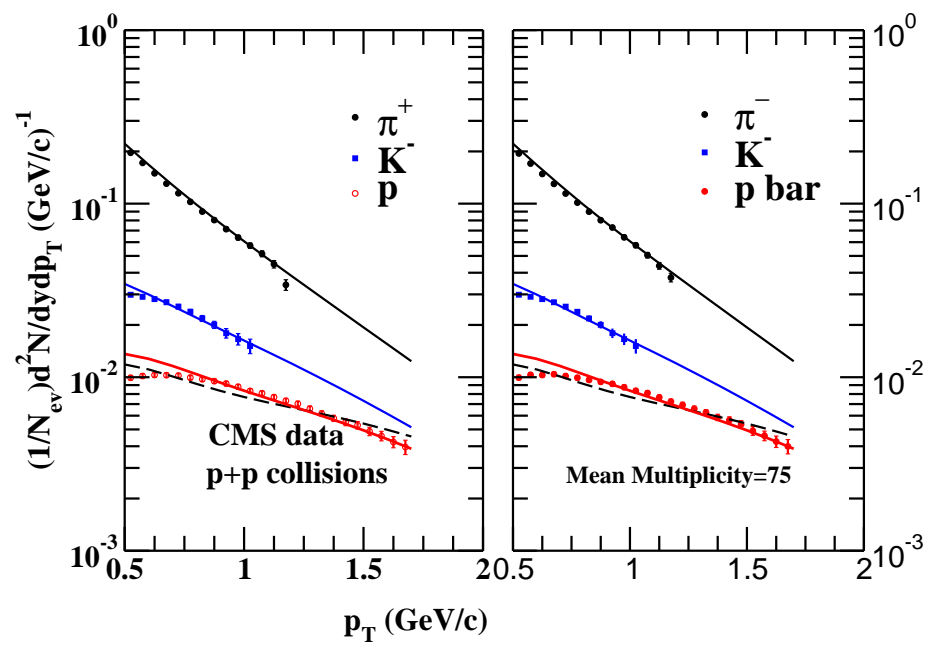

Figure 3: The transverse momentum spectra of identified charged particles such as pions, kaons and protons with mean multiplicity $=75$ have been plotted for $\sqrt{s_{N N}}=7 \mathrm{TeV}$, LHC energy. The solid circles represent the data points observed by CMS collaboration. Here, the left panel is for $\pi^{+}, K^{+}, p$ and the right panel is for $\pi^{-}, K^{-}$and $\bar{p}$. The solid lines are theoretical calculations.

\section{Summary}

The same data are explained by using blast wave (BGBW) models in [1], where the authors extract a common flow velocity $\beta$ and common kinetic temperature $T_{k i n}$ using simultaneous fit. The authors also hint for the presence of collectivity from the BGBW analysis.

The authors in [4] explains the pseudo rapidity distribution of charged hadrons with initial energy density larger than the critical energy density estimated with improved Bjorken estimation. The application of hydrodynamics explaining the pseudo rapidity distribution already establishes the collectivity in the medium formed in $p+p$ collisions at $\sqrt{s_{N N}}=7 \mathrm{TeV}$ LHC energy. In this hydrodynamic description, we also find the total charged hadron spectra and individual pion and kaon spectra are explained well. The explaination using hydrodynamic prescription implies the presence of collectivity in $p+p$ collisions at 7TeV, LHC energy. The critical initial energy density required to explain the data is more than the critical value for QGP formation for the above discussed EOS.

\section{References}

[1] P. Ghosh, S. Muhuri, J. K. Nayak, R. Varma, J. Phys. G 41035106 (2014).

[2] V. Khachatryan et al., for CMS collaboration, J. High. Energy. Phys. 09, 091 (2010).

[3] L. Van Hove, et al., Phys. Lett. B18, 138(1982); Y. Hama and S. S. Padula, Phys. Rev. D. 37, 3237 (1988); P. Levai and B. Muller, Phys. Rev. Lett. 67, 1519 (1991); T. Alexopoulos et al., Phys. Lett. B528, 43(2002); S. K. Prasad et al., Phys. Rev. C 82, 024909 (2010); J. Casalderry-Solana and U. A. Weidemann, Phys. Rev. Lett.104, 102301 (2010); D.d'Enterria et al., Eur. Phys. J. C 66, 173 (2010); P. Bozek, Eur. Phys. J. C 71, 1530 (2011); K. Werner, L. Karpenko and T. Pierog, Phys. Rev. Lett. 106, 12204 (2011); F. M. Liu and K. Werner, Phys. Rev. Lett. 106, 242301 (2011); V. Topor Pop, M. 
Gyulassy, J. Barrette, C. Gale and A. Warburton, Phys. Rev. C 86, 044902 (2012); T. Kalaydzhyan, Edward Shuryak, arXiv:1503.05213; P. Romatschke, arXiv:1504.02529.

[4] M. Csanad and T. Csorgo, arxiv:1307.2082 (2013);

[5] K. Aamodt et al., for ALICE collaboration, Phys. Rev. D 84, 112004 (2011).

[6] V. Khachatryan et al., for CMS collaboration, J. High. Energy. Phys. 05, 029 (2011).

[7] J. Adams et al., STAR collaboration, Phys. Rev. Lett. 95, 152301 (2005).

[8] J. Adams et al., STAR collaboration, Phys. Rev. C. 71, 044906 (2005).

[9] V. Khachatryan et al. Phys. Rev. Lett. 106, 082001 (2011).

[10] J. D. Bjorken, Phys. Rev. D, 27, 140 (1983).

[11] J. K. Nayak, J. Alam, S. Sarkar and B. Sinha, J. Phys. G, 35,104161 (2008).

[12] P. Mohanty, J. K. Nayak, J. Alam and S. Das, Phys. ReV. C,82 (2010) 034901.

[13] Jajati K. Nayak, J. Alam, T. Hirano, Sourav Sarkar and B. Sinha, Phys. ReV. C 85064906 (2012).

[14] S. Sarkar, S. Ghosh, J. Phys. Conf. Ser. 374, (2012)012010.

[15] J. K. Nayak, B. Sinha, Phys. Lett. B 719(2013)110.

[16] M. Asakawa and T. Hatsuda, Phys. Rev. D, 55, 4488 (1997).

[17] S. Gupta, X. Luo, B. Mohanty, H. G. Ritter and N. Xu, Science 332, 1525(2011).

[18] V. Khachatryan et al., For CMS collaboration, Eur. Phys. J. C, 72, 2164 (2012).

[19] F. Cooper and G. Frye, Phys. Rev. D. 10, 186 (1974).

[20] A. Andronic it et al., Nucl. Phys. A. 904-905, 535c (2013).

[21] A. Andronic it et al., arxiv:1311.4662 (2013), to be published in JPG.

[22] M. D. Azmi and J. Cleymans, arxiv:1310.0217 (2013). 